\title{
РАВНОВЕСИЕ ИОННОГО ОБМЕНА НА ОКСИГИДРАТНЫХ СОРБЕНТАХ, ОСЛОЖНЕННОЕ ДИСПЕРСИЕЙ ОБМЕННЫХ ЦЕНТРОВ ПО КОНСТАНТЕ ОБМЕНА
}

\section{Кудрявцев П. Г.}

\section{ВВЕДЕНИЕ}

Оксигидраты металлов представляют собой неорганические полимерные трехмерные наноструктурированные материалы. В последние годы они находят все большее применение в различных областях современных высоких технологий: в качестве сорбентов, носителей для катализаторов, наполнителей в полимерах, связующих в неорганических композитах. Их функциональные свойства во многом определяются характером поверхностных активных центров. Так, с этим явлением связывается непостоянство констант обмена в зависимости от степени их замещения. В литературе неоднократно обсуждалась причина неоднородности активных центров на поверхности оксигидратов металлов - неорганических полимеров, в т. ч. получаемых золь-гель. Так, с этим явлением связывалось непостоянство константы обмена у неорганических сорбентов в зависимости от степени обмена ${ }^{1}$.

Неоднородность активных центров оксигидратов может быть обусловлена химическими факторами, например, наличием в структуре ионов гидроксония, гидроксо-, оксо- или оловых групп, а также различным образом координированной воды. С другой стороны, активные центры и функциональные группы одной и той же химической природы могут иметь различные характеристики, обусловленные физической неоднородностью материала и их ближайшим окружением. Так, в кристаллических оксигидратах одни и те же функциональные группы могут располагаться в различных кристаллографических позициях и на различных местах поверхности кристаллитов, что обусловливает их энергетическую неоднородность ${ }^{2}$. Кроме этого, неоднородность активных центров может быть

1 Кокотов Ю.А, Пасечник В.А. Равновесие и кинетика ионного обмена. Ленинград : Химия, 1970. С. 108.

2 Белинская Ф.А. Неорганические иониты. Иониты в химической технологии. Ленинград, 1982. С. 158-203. 
обусловлена взаимным влиянием сорбируемых ионов при их размещении в матрице ионообменника ${ }^{3}$.

Такое обилие различных факторов, оказывающих влияние на константы сорбции и ионного обмена, и приводят к значительным отклонениям их значений в обе стороны от некоторой идеальной величины. Таким образом, у оксигидратных материалов спектр активных центров не носит вид $\delta$-функции и представляет собой некоторое распределение, часто полимодального характера. Этот эффект наблюдается при анализе данных по потенциометрическому титрованию в некоторых оксигидратах и выражается в сложном асимметричном характере перегибов на кривых потенциометрического титрования. Так, у оксигидрата титана (IV) вообще не представляется возможным выделить отдельные центры, что выражается в различной их оценке у разных авторов ${ }^{4}$. У гидратированных оксидов $\mathrm{MnO}_{2}^{5}$ и $\mathrm{Nb}_{2} \mathrm{O}_{5}{ }^{6}$ эти перегибы на кривых потенциометрического титрования более четко выражены, чем у $\mathrm{TiO}_{2}$, но их симметрия далека от идеальной. В связи с этим становится понятным, почему при описании сорбционного равновесия на оксигидратных материалах с помощью дискретных зависимостей хорошие результаты получаются лишь при неограниченном возрастании количества активных центров. Однако для описания ионообменного равновесия на оксигидратных ионообменниках применяют преимущественно дискретные зависимости, такие, как модель полифункционального ионита ${ }^{7}$ или модель ступенчатого обмена ${ }^{8}$. В некоторых случаях эти модели хорошо описывают истинные зависимости только при неограниченном возрастании числа сортов обменных центров или ступеней обмена. В работах ${ }^{9}$ была сделана попытка определения функции распределения

3 Ласкорин Б.Н., Стрелко В.В., Стражеско Д.Н., Денисов В.И. Сорбенты на основе силикагеля в радиохимии. Химические свойства. Применение. Москва : Атомиздат, 1977. 304 с.

${ }^{4}$ Вольхин В.В., Онорин С.А. Изв. АН СССР. Неорганические материалы. 1976. T. 12. № 8. C. 14-15.

Онорин С.А., Вольхин В.В., Змльберман М.В., Ходяшев Н.Б. Синтез катионитов с повышенной обменной емкостью. Известия AH CCCP. Неорганические материаль. 1978. Т. 14. С. 150-153.

Кудрявцев П.Г., Онорин С.А., Вольхин В.В. Неорганический ионообменник ИСН-1, селективный к ионам лития. Изв. ВУЗ. Цветная металлургия. 1977. № 3. C. $50-53$.

7 Белинская Ф.А. Неорганические иониты. Иониты в химической технологии. Ленинград, 1982. С. 158-203.

Белинская Ф.А. Ступенчатое ионообменное равновесие. Модельные представления. Вестник ЛГУ. 1983. № 22. С. 42-48.

Бобыренко Ю.Я. Распределение кислотных и основных групп поверхности рутила по величине констант диссоциации. Журнал физической химии. 1978. Т. 92. № 56. С. 1488-1490; Бобыренко Ю.Я. О потенциометрическом методе определения электроповерхностных свойств дисперсных материалов. Коллоидный журнал. Т. 48. № 4. C. 649-653. 
обменных центров по константе обмена для гидратированного $\mathrm{TiO}_{2}$. Однако предложенная методика носила приближенный характер, а некоторые предположения не имели строгого доказательства.

В связи с этим в данной работе была предпринята попытка построения кривой потенциометрического титрования на основе функции распределения обменных центров по константе обмена. Функция распределения обменных центров по константе обмена была предложена в работах $^{10}$ для описания изотермы обмена на органических ионитах, а в работе ${ }^{11}$ - для равновесия обмена на неорганических оксигидратных сорбентах.

Рассмотрим процесс катионного обмена (для анионного обмена функциональные зависимости подобны):

$$
M e^{+}+\bar{H}^{+} \rightarrow H^{+}+\overline{M e}^{+}
$$

Для количественного описания ионообменного равновесия обычно применяют уравнение изотермы обмена в форме закона действующих масс $^{12}$. Изотерма обмена - это зависимость эквивалентной доли одного из обменивающихся ионов в твердой фазе $\left(E_{a}\right)$ от эквивалентной доли этого же иона в растворе $\left(a_{A}\right)$. В общем виде это уравнение можно записать в следующей форме:

$$
K=\frac{E_{B}^{1 / z_{B}} a_{A}^{1 / z_{A}}}{E_{A}^{1 / z_{A}} a_{B}^{1 / z_{B}}}
$$

где: $K$ - коэффициент равновесия (константа обмена): $E_{A}$ и $E_{B}-$ концентрации противоионов А А и В в фазе ионита; $a_{A}$ и $a_{B}$ активности противоионов в растворе.

Условиями применимости (2) являются равноценность всех связей каждого из двух обменивающихся ионов во всей массе ионита, т. е. условие монофункциональности ионита, а также независимость коэффициентов активности ионов $a_{A}$ и $a_{B}$ в твердой фазе от степени замещения ${ }^{13}$.

${ }^{10}$ Рогинский С.3. Гетерогенный катализ. Некоторые вопросы теории. Москва : Наука, 1979.

${ }_{11}$ Кудрявцев П.Г. Равновесие ионного обмена на оксигидратных сорбентах, осложненное дисперсией обменных центров по константе обмена. Журнал физ. Химии. 1987. Т. 61. № 3. С. 848-851.

12 Кокотов Ю.А, Пасечник В.А. Равновесие и кинетика ионного обмена. Лениград : Химия, 1970. С. 108; Никольский Б.П., Парамонова В.И. Законы обмена ионов между твердой фазой и раствором. Усn. хим. 1939. Т. 8. С. 1535-1537.

13 Никольский Б.П. О классификации ионитов в свете современной теории обмена ионов. Хроматография. Ленинград, 1956. С. 5-15. 
Для более строго описания равновесия ионного обмена Гельферихом ${ }^{14}$ был предложен способ расчета коэффициентов активности в твердой фазе и термодинамических констант обмена, в котором ионообменник рассматривался как твердый раствор. Используя этот подход, уравнение (2) стали широко применять для описания обмена на различных ионитах, на которых обмен протекает без перестройки кристаллической структуры полимерного каркаса. При таком подходе в коэффициенты активности формально включаются все факторы, приводящие к отклонению экспериментальных данных от выполнения уравнения (2).

Однако, на наш взгляд, более полезными для понимания характера взаимодействий в твердой фазе являются анализ изменений и выявление причин этих изменений, поиски других форм функциональной зависимости, отвечающих уравнению (2), а в более общем виде - уравнению (28).

Как известно, растворы, твердые тела и такие системы, как неорганические ионообменные материалы и процессы, протекающие в них, не поддаются описанию посредством одних только законов динамики и электростатики. В этих системах при ионном обмене протекают процессы, ход которых невозможно предсказать совершенно точно. Поэтому подобные процессы называются случайными, а их структуры, в свою очередь, также носят случайный характер. Здесь случайность проявляется как следствие неполноты знаний исследователя обо всех деталях исследуемой системы, о ее прошлом и о воздействиях, которым она подвергается в результате протекающих в ней процессов. Другим факторам случайность может быть присуща в силу их физической природы. В любом случае для описания случайных явлений следует применять статистические методы. В частности, это относится К неорганическим ионообменным материалам как макроскопическим системам, состоящим из очень большого числа атомов, молекулярных образований и структурных элементов. Теоретически возможно записать в символической форме уравнения, описывающие поведение всех элементов, входящих в состав макросистемы, с учетом всех действующих на них сил. Но из-за многочисленности этих уравнений их решению препятствуют непреодолимые математические трудности. Поэтому для количественного описания данной системы необходимо использовать методы, основанные на понятии вероятности, с использованием статистического подхода.

\footnotetext{
${ }^{14}$ Гельферих Ф. Иониты. Издательство иностранной литературы. Москва, 1962. $490 \mathrm{c}$.
} 


\section{1. Причины возникновения неоднородности обменных центров}

у неорганических ионообменных материалов

Обычно значение константы диссоциации $K_{a}$ уменьшается с увеличением степени замещения одних ионов в ионообменнике на другие ионы даже в случае обмена ионов с одинаковыми зарядами, когда исключен эффект зарядовой селективности. Часты случаи, когда для монофункциональных ионитов наблюдаются более сложные зависимости. Соответственно, на изотермах обмена выявляются несимметричность, точки перегиба, пологие участки, характерные для обмена на полифункциональных ионитах ${ }^{15}$.

Такое проявление полифункциональности для органических ионитов принято объяснять возникновением химической неоднородности, обусловленной технологическими отклонениями при их синтезе $\mathrm{e}^{16}$. Для неорганических ионитов проявление полифункциональности связывают с содержанием групп различной природы, с существованием нескольких неравноценных позиций расположения противоионов в кристаллической структуре ионообменного материала ${ }^{17}$. В связи с этим была достаточно подробно разработана термодинамика обмена ионов на полифункциональных ионитах $^{18}$. В литературе описаны две основных модели возникновения полифункциональности в неорганических ионообменных материалах. Первая представляет собой модель полифункционального ионообменника, а вторая носит название модели ступенчатого обмена.

В соответствии с моделью полифункционального ионообменника он имеет $n$-сортов активных групп или активных центров, последовательность участия которых в обмене определяется значениями частных констант обмена. Для случая реакции, соответствующей уравнению (1), это константы кислотности обменных

${ }^{15}$ Кокотов Ю.А., Пасечник В.А. Равновесие и кинетика ионного обмена. Ленинград : Химия, 1970. С. 108; Белинская Ф.А. Неорганические иониты. Иониты в химической технологии. Ленинград, 1982. С. 158-203; Гельферих Ф. Иониты. Издательство иностранной литературы. Москва, 1962. 490 с.; Ионный обмен / под ред. Я. Маринского. Москва : Мир, 1968. 565 с.; Самсонов Г.В. Термодинамические, кинетические и динамические особенности ионного обмена с участием ионов органических веществ. Ионный обмен. Ленинград, 1981. С. 126-137; Челищев Н.Ф. Ионообменные свойства минералов / АН СССР, Институт минералогии, геохимии и кристаллохимии редких элементов. Москва : Наука, 1973. 203 с.; Гриссбах Р. Теория и практика ионного обмена. Москва : Издательство иностранной литературы, 1963. 499 с.; Белинская Ф.А., Милицина Э.А. Неорганические ионообменные материалы на основе труднорастворимых соединений сурьмы (V). Успехи химии. 1980. T. 49. № 10. C. 1904 1936; Брек Д. Цеолитовые молекулярные сита. Ленинград, 1976. 781 с.

16 Белинская Ф.А. Неорганические иониты. Иониты в химической технологии. Ленинград, 1982. С. 158-203; Ионный обмен / под ред. Я. Маринского. Москва : Мир, 1968, 565 с.

18 Брек Д. Цеолитовые молекулярные сита. Ленинград, 1976. 781 с.

18 Белинская Ф.А. Неорганические иониты. Иониты в химической технологии. Ленинград, 1982. С. 158-203. 
центров. Модель полифункционального ионообменника позволяет описать прямолинейные, криволинейные и ступенчатые зависимости $p K_{a}=-\lg \left(K_{a}\right)$ от $\mathrm{pH}$. Модель полифункционального ионообменника это модель параллельных реакций на энергетически неравноценных активных центрах и в принципе не может объяснить некоторых особенностей изменения $K_{a}$ в зависимости от степени замещения ${ }^{19}$.

В модели ступенчатого обмена ионообменник рассматривается как комплексное соединение $\mathrm{RA}_{\mathrm{n}}$, где $n$ соответствует числу последовательных стадий замещения лигандов в координационной сфере. В качестве примеров реализации обоих подходов можно привести работы по исследованию ионообменных свойств кристаллической сурьмяной кислоты ${ }^{20}$. Модель полифункционального ионообменника была привлечена для описания ступенчатого обмена на кристаллической сурьмяной кислоте ${ }^{21}$. Однако более точные результаты и физическую сущность механизма обмена ионов на кристаллической сурьмяной кислоте, как было показано, отражает модель ступенчатого обмена 22 .

В качестве другого примера можно привести гидратированный пентаоксид ниобия (V), у которого было обнаружено 4 типа ионообменных центров ${ }^{23}$. Кривые потенциометрического титрования для исходного и модифицированного различными добавками гидратированного пентаоксида ниобия (V) представлены на рис. 1.

Механизм работы этих обменных центров был различный. Базируясь на основе представлений о структуре данного гидратированного оксида ${ }^{24}$, были показаны причины обнаруженных различий в свойствах первых трех обменных центров, к которым относятся различные по кислотности ионообменные ОН-группы с $\mathrm{pK}_{1}=5,7 \pm 0,5, \mathrm{pK}_{2}=6,7 \pm 0.5$ и $\mathrm{pK}_{3}=10.3 \pm 0.2$.

19 Кокотов Ю.А., Пасечник В.А. Равновесие и кинетика ионного обмена. Ленинград : Химия, 1970. С. 108.

${ }^{20}$ Белинская Ф.А. Неорганические иониты. Иониты в химической технологии. Ленинград, 1982. С. 158-203; Белинская Ф.А. Ступенчатое ионообменное равновесие. Модельные представления. Вестник ЛГУ. 1983. № 22. С. 42-48; Белинская Ф.А., Милицина Э.А. Неорганические ионообменные материалы на основе труднорастворимых соединений сурьмы (V). Успехи химии. 1980. Т. 49. № 10. С. 1904-1936.

21 Белинская Ф.А. Ступенчатое ионообменное равновесие. Модельные представления. Вестник ЛГУ. 1983. № 22. С. 42-48.

${ }_{22}$ Белинская Ф.А., Милицина Э.А. Неорганические ионообменные материалы на основе труднорастворимых соединений сурьмы (V). Успехи химии. 1980. Т. 49. № 10. С. $1904-1936$.

Кудрявцев П.Г. Онорин С.А. Сорбция ионов щелочных металлов гидратированной пятиокисью ниобия. Неорганические ионообменники (синтез, структура, свойства). 1977. № 212. С. 105-108.

Кудрявцев П.Г., Онорин С.А., Вольхин В.В. Неорганический ионообменник ИСН-1, селективный к ионам лития. Изв. ВУЗ. Цветная металлургия. 1977. № 3. C. $50-53$. 


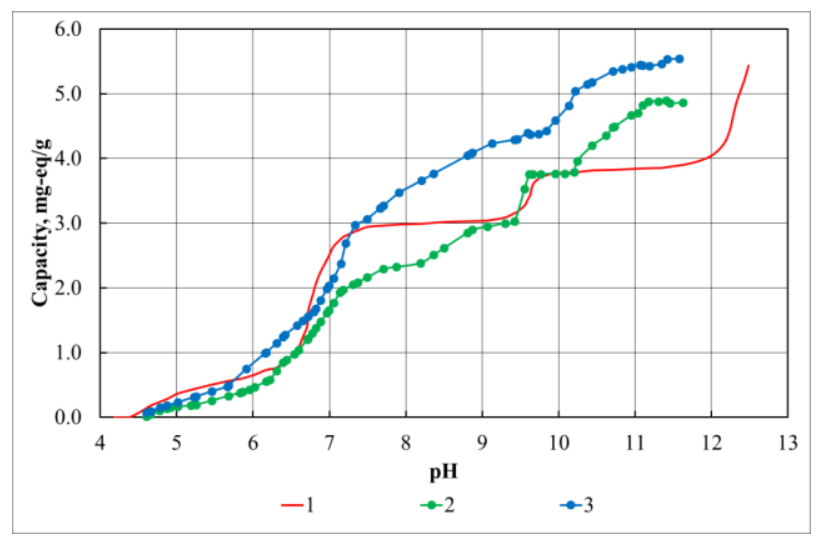

Рис. 1. Кривые потенциометрического титрования гидратированного пентаоксида ниобия (V) (ГПН), модифицированного различными добавками: 1 - исходный ГПН; 2 - ГПН с добавкой Fe(III); 3 - ГПН с добавкой Мо(VI)

При их диссоциации у полианионного каркаса гидратированного оксида возникает отрицательный заряд, который компенсируется ионами гидроксония $\mathrm{H}_{3} \mathrm{O}^{+}$, расположенными в его структуре. При ионном обмене на ионы щелочных металлов для компенсации всего избыточного отрицательного заряда полианионного каркаса оксида катионами обменная емкость гидратированного пентаоксида ниобия (V) достигает величины 1 мг-экв/мг-моль $\mathrm{Nb}_{2} \mathrm{O}_{5}$. Для обнаруженного структурного мотива реализация первого обменного центра соответствует заполнению каждого 4 слоя из полостей в кристаллической структуре материала. Далее заполняются левый и правый слои, соседние с заполненным, и уже затем произойдет заполнение последнего из четырех слоев.

Поскольку все слои одинаковые между собой, то неодновременность их заполнения может быть связана только с их взаимным влиянием и обусловлена индукционным эффектом, возникающим при делокализации электронов ${ }^{25}$. В данном случае делокализация электронов проявляется как следствие $(p \rightarrow d)_{\pi}$ сопряжений, возникающих при взаимодействии незаполненных подуровней атомов ниобия и $p$-подуровней атомов кислорода. При замещении атомов водорода атомами щелочных металлов вследствие существенно электронно-донорных свойств последних 1962.

${ }^{25}$ Mortimer C.T. Reactions heats and bonds strengths. Oxford : Pergamon Prass, 
будет наблюдаться индукционный эффект, который направлен в сторону увеличения электронной плотности на связи О-Н функциональных групп, расположенных в соседних с заполненным слоем. Следствием наложения воздействий каждого четвертого слоя энергетически выгодным становится заполнение соседних с ним слоев и уже только потом - последнего слоя.

При реализации в ходе обмена центров с $\mathrm{pK}_{4}=11,6 \pm 0,4$ сорбция ионов щелочных металлов происходит также за счет процесса деоксоляции некоторой части оксо-связей в структуре гидратированного пентаоксида ниобия (V). Полости в структуре фазы гидратированного пентаоксида ниобия (V) имеют такую конфигурацию, что обеспечивают находящимся в них ионам щелочных металлов октаэдрическое окружение со стороны атомов кислорода октаэдров $\mathrm{NbO}_{6}$. Молекулы воды и ионы гидроксония $\mathrm{H}_{3} \mathrm{O}^{+}$могут располагаться в оставшихся свободных пространствах. Приведенное выше рассмотрение различия обменных центров было основано на модели с неограниченными размерами частиц реагирующего вещества. Однако в реальных объектах их размеры будут вносить соответствующее искажение в идеальную картину.

Было обнаружено, например, что для гидратированного оксида алюминия наблюдается некоторое увеличение $\mathrm{pK}_{\mathrm{a}} \mathrm{OH}-г р у п п ~$ с уменьшением ближнего порядка ${ }^{26}$. Этот эффект вполне объясняется с позиций конечности размеров частиц гидратированных оксидов. Такие частицы можно интерпретировать с кристаллографических позиций как области когерентного рассеяния (блоки), имеющие очень малые размеры, меньше 2.0 нм. Они и обусловливает наличие ближнего порядка. Строго говоря, эти частицы не являются изолированными агломератами. Полимерные цепи могут распространяться на несколько частиц, претерпевая на их границе искажения различного типа, например, разветвления, изгиба или обрыва.

На концах полимерных цепей кислотность ОН-групп будет отличаться от кислотности групп в центре. Концевые группы будут близки по $\mathrm{pK}_{a}$ к ОН-группам изолированных мономерных частиц, так как воздействие на эти группы катионов гидратированного оксида через делокализованные $(p \rightarrow d)_{\pi}$ сопряжения орбиталей будет значительно ниже, чем на группы в центре частиц. Аналогичные эффекты наблюдаются, например, в случае силикагеля, в котором с повышением степени полимеризации уменьшались значения $\mathrm{pK}_{a}$

26 Кудрявцев П.Г. Онорин С.А., Вольхин В.В. Структурообразование и ионообменные свойства смешанных оксидов в системе $\mathrm{Li}_{2} \mathrm{O}-\mathrm{Fe}_{2} \mathrm{O}_{3}-\mathrm{Nb}_{2} \mathrm{O}_{5}$. Известия АН СССР. Серия Неорганические материальы. Т. 27. № 7. 1991. С. 1479-1482. 
гидроксильных групп ${ }^{27}$. Рассчитанное распределение ОН-групп по величине $p K_{a}$ в поперечнике первичной частицы гидроксида, для частиц различного размера, будет иметь вид, представленный на рис. 2. Из этого рисунка видно, что с уменьшением размера частиц будет происходить увеличение средней по всем ОН-группам частицы величины рК.

Можно ожидать, что в экспериментах по потенциометрическому титрованию измеряется не истинная величина $\mathrm{pK}$, а некоторая эффективная величина, соответствующая средней для всей частицы. Ее значение можно определить как средне интегральную величину:

$$
p K_{\mathrm{cp}}\left(R_{0}\right)=\frac{1}{R_{0}} \int_{0}^{R_{0}} p K(l) d l
$$

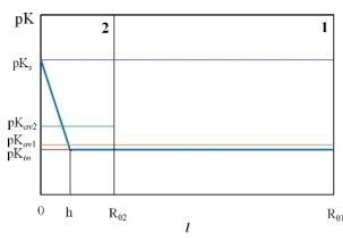

a

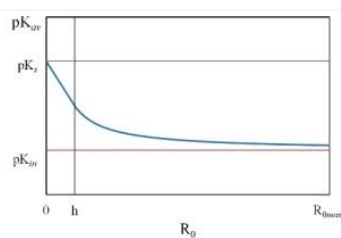

b

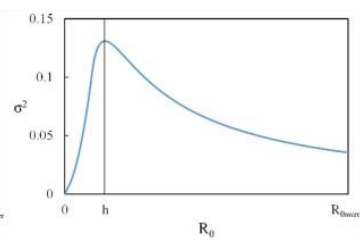

C

Рис. 2. К выводу уравнений (3) и (4). $a$ - распределение величины константы диссоциации обменных ОН-групп неорганического оксигидратного ионообменника по сечению частиц кристаллитов разных размеров (1 - болышой кристаллит, 2 - малый кристаллит, $\left.\boldsymbol{R}_{02}<\boldsymbol{R}_{01}\right) ; \boldsymbol{b}$ - зависимость среднего значения константы диссоциации обменных ОН-групп в зависимости от размера частицы кристаллита; $c$ - зависимость дисперсии $\left(\sigma^{2}-\right.$ квадрат стандартного отклонения) значения константы диссоциации обменных ОН-групा в отдельном кристаллите в зависимости от его размеров $\left(\boldsymbol{R}_{0}\right) ; \mathbf{p K}_{s}$ - значение константы диссоциации обменных ОН-групп ионообменника на поверхности частицы кристаллита; pK $_{\text {in }}$ - значение константы диссоциации обменных ОН-групп ионообменника на внутри объема частицы кристаллита; $\mathbf{p K}_{a v 1}$ и $\mathbf{p K}_{a v 2}$ - значение средних констант диссоциации обменных ОН-групп ионообменника, усредненные по объему соответствующей частицы кристаллита

27 Ласкорин Б.Н., Стрелко В.В., Стражеско Д.Н., Денисов В.И. Сорбенты на основе силикагеля в радиохимии. Химические свойства. Применение. Москва : Атомиздат, 1977. $304 \mathrm{c}$. 
График функции (3) будет иметь вид, представленный на рис. 2b. То есть с ростом размера частиц будет происходить уменьшение значения $\mathrm{pK}$, усредненного по всему объему частицы. С ростом размера частиц эта функция будут стремиться асимптотически к значению $\mathrm{pK}_{i n}$, соответствующему рК внутри частицы.

При этом среднеквадратичное отклонение $\left(\sigma^{2}\right)$ истинных значений рК от средней величины будет равно:

$$
\sigma^{2}\left(R_{0}\right)=\frac{1}{R_{0}} \int_{0}^{R_{0}}\left(p K(l)-p K_{\mathrm{cp}}\left(R_{0}\right)\right)^{2} d l
$$

После преобразования и с учетом (3) получим:

$$
\sigma^{2}\left(R_{0}\right)=\frac{1}{R_{0}} \int_{0}^{R_{0}}(p K(l))^{2} d l-\frac{1}{R_{0}^{2}}\left(\int_{0}^{R_{0}} p K(l) d l\right)^{2}
$$

График функции (5) имеет вид, представленный на рис. 2c. Из рисунка следует, что при малых размерах частиц в гидратированном оксиде будет наблюдаться значительный разброс истинных значений $p K$ от средней величины, что должно отразиться на кривой потенциометрического титрования. Она должна стать очень пологой без резких волн.

Такой эффект наблюдается на кривых титрования гидратированного диоксида титана ${ }^{28}$. В отличие от кривых титрования гидратированного пентаоксида ниобия $(\mathrm{V})^{29}$ и гидратированного оксида алюминия ${ }^{30}$, кривые титрования гидратированного диоксида титана более пологи и представляют собой монотонно возрастающие зависимости ${ }^{31}$. Эксперименты по диффузному рассеянию рентгеновских лучей показали наличие хорошо выраженного ближнего порядка в гидратированном оксиде алюминия и гидратированном пентаоксида

28 Онорин С.А., Вольхин В.В., Змльберман М.В., Ходяшев Н.Б. Синтез катионитов с повышенной обменной емкостью. Известия AH CCCP. Неорганические материальл. 1978. Т. 14. С. 150-153.

${ }_{29}$ Кудрявцев П.Г., Онорин С.А., Вольхин В.В. Неорганический ионообменник ИСН-1, селективный к ионам лития. Изв. ВУЗ. Цветная металлургия. 1977. № 3. C. 50-53; Кудрявцев П.Г., Онорин С.А. Сорбция ионов щелочных металлов гидратированной пятиокисью ниобия. Неорганические ионообменники (синтез, структура, свойства). 1977. № 212. С. 105-108.

${ }^{30}$ Кудрявцев П.Г., Онорин С.А., Вольхин В.В. Влияние условий осаждения на строение и ионообменные свойства гидратированного оксида алюминия. Известия ВУЗ. Цветная металлургия. 1990. № 2. С. 21-26.

Кудрявцев П.Г., Онорин С.А. Сорбция ионов щелочных металлов гидратированной пятиокисью ниобия. Неорганические ионообменники (синтез, структура, свойства). 1977. № 212. С. 105-108. 
ниобия (V) и практически полное его отсутствие в гидратированном диоксиде титана ${ }^{32}$. Следовательно, вывод о связи кислотно-основных свойств гидратированных оксидов с размерами области упорядоченности в их структурах является достаточно обоснованным.

Отсюда открывается один из путей регулирования ионообменных свойств оксигидратных сорбентов за счет изменения продолжительности участков ближнего порядка в их структурах. Размеры области упорядочения можно регулировать изменением $\mathrm{pH}$ осаждения и порядка сливания реагентов при синтезе оксигидратов, а также введением в их состав ионов добавок.

\section{2. Статистический подход к описанию ионообменного равновесия на неорганических ионообменных материалах}

Обычно вероятность того или иного состояния системы определяют при помощи статистического ансамбля, который представляет собой совокупность большого числа воображаемых или действительных элементов исследуемой системы. В нашем случае сорбционные центры, имеющиеся в составе неорганических ионообменных материалов, представляют именно такой статистический ансамбль. Так в одном грамме неорганического ионообменного материала может содержаться более $10^{21}$ сорбционных центров, поэтому статистический подход к описанию ионообменных процессов вполне приемлем.

Ранее уже делались попытки описания ионообменных равновесий с использованием методов статистической термодинамики ${ }^{33}$. Так, для слабокислотного катионита классической теорией устанавливается точное соотношение между степенью диссоциации и значениями $\mathrm{pH}$ в ионите и растворе. Использование методов статистической

32 Кудрявцев П.Г., Онорин С.А. Сорбция ионов щелочных металлов гидратированной пятиокисью ниобия. Неорганические ионообменники (синтез, структура, свойства). 1977. № 212. С. 105-108; Кудрявцев П.Г., Онорин С.А., Вольхин В.В. Влияние термообработки на ионообменные свойства и строение материалов, содержащих гидратированный оксид алюминия. Известия $B У 3$. Цветная металлургия. 1990. № 3. С. 26-31; Кудрявцев П.Г., Онорин С.А., Вольхин В.В. Структурообразование и ионообменные свойства смешанных оксидов в системе $\mathrm{Li}_{2} \mathrm{O}-\mathrm{Fe}_{2} \mathrm{O}_{3}-\mathrm{Nb}_{2} \mathrm{O}_{5}$. Известия АН СССР. Сер. Неорганические материальл. 1991. Т. 27. № 7. С. 1479-1482; Кудрявцев П.Г., Онорин С.А., Вольхин В.В. Структурообразование и ионообменные свойства смешанных оксидов в системе $\mathrm{Li}_{2} \mathrm{O}-\mathrm{Fe}_{2} \mathrm{O}_{3}-\mathrm{Nb}_{2} \mathrm{O}_{5}$. Известия АН СССР. Серия Неорганические материаль. 1991. Т. 27. № 7. С. 1479-1482; Кудрявцев П.Г., Онорин С.А., Вольхин В.В., Ходяшев Н.Б., Ходяшев М.Б., Вольхин Д.В. Неорганические сорбенты на основе гидроксидов металлов для извлечения микропримесей из жидких сред. Сб. научных трудов. Химия, технология, промышленная экология неорганических соединений. Пермь, 1998. Вып. 1. С. 101-106.

${ }_{33}$ Гельферих Ф. Иониты. Издательство иностранной литературы. Москва, 1962. 490 с.; Никольский Б.П. (ред.) Физическая химия. Теоретическое и практическое руководство. Ленинград : Химия, 1987. 880 с. 
термодинамики в основном сводилось к введению поправочного члена в уравнение изотермы ионного обмена.

Рассмотрим статистический ансамбль, состоящий из $N$ одинаковых дискретных активных центров в неорганическом ионообменнике, на каждом из которых протекает случайный процесс обмена противоионов. Каждый активный центр может находиться в множестве различных состояний, количество которых равно $\boldsymbol{i}$. Пусть в некоторый момент времени $\boldsymbol{t}$ среди всех центров этого ансамбля в состоянии с номером $\boldsymbol{i}$ находится некоторое количество центров, равное $N_{i}(t)$. Очевидно, что сумма всех этих чисел равна числу центров в ансамбле:

$$
\sum_{i} N_{i}(t)=N
$$

Вероятность того, что один какой-то центр ансамбля в момент времени $\boldsymbol{t}$ находится в $\boldsymbol{i}$-ом состоянии, равна следующей величине:

$$
W_{i}(t)=\lim _{N \rightarrow \infty} \frac{N_{i}(t)}{N}
$$

Таким образом, вероятность равна пределу, к которому стремится отношение числа $N_{i}(t)$ к числу $N$ центров в ансамбле, когда последнее неограниченно возрастает. Зависимость вероятности (6) от номера состояния или от какой-либо другой величины, например, константы диссоциации соответствующего центра, которая однозначно определяет состояние системы, называется функцией распределения систем ансамбля по состояниям.

Просуммируем обе части равенства (7) по всем возможным значениям номера $\boldsymbol{i}$. C учетом (6) получим условие нормировки вероятности:

$$
\sum_{i} W_{i}=1
$$

Зная эту функцию, можно вычислить вероятность, с которой система окажется в том или ином состоянии в заданный момент времени. Нестационарные состояния ионообменной системы определяют кинетику процесса ионного обмена. Конечной стадией кинетических изменений в ионообменном процессе является равновесное состояние системы. В равновесном состоянии системы случайный процесс становится стационарным, это означает, что его характер не изменяется с течением времени. Такой процесс может протекать в любой равновесной макроскопической системе, которая время от времени переходит из одного микроскопического состояния 
в другое, при неизменном общем макроскопическом состоянии. Для стационарного случайного процесса вероятность $W_{i}$ не зависит от времени.

Для стационарного случайного процесса существует определенная вероятность, равная $\widetilde{W}_{i}$, которая соответствует тому, что данная система в какой-то момент времени окажется в $i$-ом состоянии. Такую вероятность можно определить как предел отношения времен $\Delta t_{i}$, в течение которого система находилась в $\boldsymbol{i}$-м состоянии, к некоторому продолжительному промежутку времени длительностью $\Delta t$, в котором наша ионообменная система будет многократно переходить из одного состояния в другое при $\Delta t \rightarrow \infty$ :

$$
\widetilde{W}_{i}(t)=\lim _{\Delta t \rightarrow \infty} \frac{\Delta t_{i}}{\Delta t}
$$

В соответствии с эргодической теоремой ${ }^{34}$, которая означает, что среднее по времени для почти всех начальных точек равно пространственному среднему, имеем:

$$
\widetilde{W}_{i}=W_{i}
$$

Реальный ионообменный материал является дискретной системой ионообменных центров, каждый из которых, кроме номера $\boldsymbol{i}$, характеризуется одной или несколькими величинами, имеющими определенный физический смысл. Например, каждый центр имеет некоторую свободную энергию $\left(\Delta G_{i}^{0}\right)$ и характеризуется определенной константной диссоциации $\left(K_{i}\right)$ или обмена:

$$
K_{i}=\exp \left(-\frac{\Delta G_{i}^{0}}{R T}\right)
$$

Обозначим константу диссоциации некоторым значением соответствующим $i$-му состоянию $-K_{i}$. Причем различным состояниям системы и, следовательно, различным номерам $\boldsymbol{i}$ может соответствовать одно и то же значение константы диссоциации $K$. Множество значений $K_{i}$, которые может принимать величина $K_{a}$, является дискретным. Поэтому эта величина, строго говоря, является дискретной случайной величиной. Совокупность всех значений величины константы диссоциации $K$ является спектром ее значений.

${ }^{34}$ Каток А.Б., Хассельблат Б. Введение в современную теорию динамических систем с обзором последних достижений / пер. с англ. под ред. А.С. Городецкого. Москва : МЦНМО, 2005. 464 с. 
Пусть среди $N$ ионообменных центров статистического ансамбля в стационарном состоянии имеется число $N(K)$ систем, для которых величина константы диссоциации принимает некоторое заданное значение $K$. Предел отношения этого числа к числу $N$ центров в ансамбле при неограниченном возрастании последнего является вероятностью $W(K)$ того, что один какой-то выбранный наугад активный центр находится в состоянии, которому соответствует значение $K$ :

$$
W(K)=\lim _{N \rightarrow \infty} \frac{N(K)}{N}
$$

Зависимость $W=W(K)$ является функцией распределения дискретной случайной величины $K$. Просуммируем обе части равенства (12) по всем возможным значениям величины $K$. Так как

$$
\sum_{K} N(K)=N
$$

Отсюда мы придем к условию нормировки вероятности:

$$
\sum_{K} W(K)=1
$$

Величина $K$ является однозначной функцией номера состояния $i$ : $K=K(i)$, т. е. каждому состоянию системы соответствует одно определенное значение $K(i)$ такой величины, как константа обмена $K$. Обратная функция $i=i(K)$, строго говоря, не является однозначной, т. е. одно и то же значение $K$ может соответствовать различным состояниям системы. Поэтому число $N(K)$ центров ансамбля, для которых величина $K$ принимает заданное значение $K$, будет связано с числом $N_{i}$ систем в $\boldsymbol{i}$-м состоянии соотношением

$$
N(K)=\sum_{i=i(K)} N_{i}
$$

где суммирование производится только по номерам состояний $i=i(K), \quad$ соответствующим заданному значению $K$. Разделив соотношение (15) на $N$, в пределе при $N \rightarrow \infty$ в соответствии с формулами (7) и (12) и используя теорему о сложении вероятностей, получим: 


$$
F(K)=\sum_{i=i(K)} W_{i}
$$

Полученная таким образом функция $F(K)$ является макроскопической функцией и имеет смысл дифференциальной функции распределения обменных центров ионообменника по константе обмена. Следует подчеркнуть еще один важный момент. Поскольку множество возможных состояний исследуемой системы при $N \rightarrow \infty$ не является счетным, то состояние такой системы можно охарактеризовать одной или несколькими величинами, которые обладают непрерывным спектром значений. В нашем случае, учитывая возможное количество активных центров, можно рассматривать спектр возможных значений константы обмена как некую непрерывную функцию. Учитывая эту функцию распределения обменных центров по константе обмена, можно записать в следующем виде:

$$
F(K)=\frac{d E}{d K}
$$

где: $d E$ - количество обменных центров с константой обмена в интервале $(K, K+d K)$.

При этом функция $F(K)$ обладает следующими свойствами:

$$
\int_{0}^{+\infty} F(K) d K=E_{0}
$$

где: $E_{0}-$ величина полной обменной емкости и является условием нормировки функции распределения. Фактически $E_{0}$ является нулевым моментом функции распределения.

$$
\begin{aligned}
& \lim _{K \rightarrow \infty} F(K)=0 \\
& \lim _{K \rightarrow 0} F(K)=0
\end{aligned}
$$

Функция $F(K)$ положительна и определена в любой точке при $K>0$. Конкретной количественной мерой формы функции распределения в математике являются моменты функции распределения. Совокупность моментов всех порядков от 0 до $\infty$ однозначно определяет распределения вероятности на ограниченном интервале. Как известно, моменты распределения являются специфической количественной мерой формы функции 
распределения ${ }^{35}$ и используются как в статистике, механике и в других отраслях науки и техники. В данном случае функция распределения представляет физическую плотность активных обменных центров в единичном объеме неорганического ионообменного материала. Таким образом, нулевой момент - это общая масса, совокупность или содержание всех обменных центров в единице объема или массы неорганического ионообменного материала. При этом каждый обменный центр характеризуется своей собственной константой диссоциации, выраженной уравнением (2).

Первый момент $\mu_{1}$ - это среднее значение, среднее или ожидаемое значение константы диссоциации для всей совокупности обменных центров. Он является мерой центра распределения вероятности, константы диссоциации, как случайной величины, характеризуемой этим распределением:

$$
\mathbb{M} K=\mu_{1}=\frac{1}{E_{0}} \int_{0}^{+\infty} K \cdot F(K) d K
$$

Второй центральный момент - это дисперсия. В теории вероятностей и статистике дисперсия является ожиданием от квадрата отклонения случайной величины от ее среднего значения. Она является мерой того, насколько далеко набор случайных значений распределен от их среднего значения. Дисперсия играет центральную роль в предложенной идее. Ее использование включает в себя описательную статистику, статистический вывод и проверку экспериментальных данных. Дисперсия является важным инструментом для статистического анализа полученных экспериментальных данных. Дисперсия представляет собой квадрат стандартного отклонения и является вторым центральным моментом распределения и ковариации случайной величины константы диссоциации с самой собой:

$$
\begin{aligned}
\mathbb{D} K=\sigma^{2}=\operatorname{Var}(K) & =\mathbb{M}\left(K-\mu_{1}\right)^{2}=\mu_{2} \\
= & \frac{1}{E_{0}} \int_{0}^{+\infty}\left(K-\mu_{1}\right)^{2} \cdot F(K) d K
\end{aligned}
$$

Центральные моменты являются более предпочтительными, чем обычные. Они вычисляются в терминах отклонений от среднего значения, а не от нуля. Это особенно касается центральных моментов высшего порядка (когда $n \geq 3$ ), поскольку они относятся только к разбросу и форме распределения, а не к его местоположению. Эти

${ }^{35}$ Spanos A. Probability theory and statistical inference. Econometric modeling with observational data. New York : Cambridge University Press, 1999. 815 p. 
моменты функции распределения для константы обмена более чувствительны к структурным аспектам неорганических ионообменных материалов.

Таким образом, третьим моментом функции распределения является асимметрия распределения:

$$
\mathbb{M}\left(K-\mu_{1}\right)^{3}=\mu_{3}=\frac{1}{E_{0}} \int_{n}^{+\infty}\left(K-\mu_{1}\right)^{3} \cdot F(K) d K
$$

Однако для использования высших моментов в качестве мер, описывающих характер распределения случайной величины, в нашем случае - констант диссоциации, их необходимо нормализовать, то есть привести к безразмерному виду. Нормализация $n$-ного центрального момента осуществляется путем его деления на величину стандартного отклонения в степени номера соответствующего момента $-\sigma^{n}$. Отсюда нормированный $n$-й центральный момент случайной величины константы диссоциации равен:

$$
\gamma_{n}=\frac{\mu_{n}}{\sigma^{n}}
$$

Эти нормированные центральные моменты являются безразмерными величинами, которые описывают распределение независимо от любого линейного изменения масштаба ${ }^{36}$. Асимметрия случайной величины $K$ - это третий стандартизированный момент $\gamma_{1}$, его также называют коэффициентом асимметрии, и он определяемый как:

$$
\gamma_{1}=\frac{\mu_{3}}{\sigma^{3}}=\frac{\mu_{3}}{\mu_{?}^{3 / 2}}
$$

Асимметрия указывает, какое направление и относительная величина того, насколько далеко распределение отклоняется от нормы. Существует два вида асимметрии распределения: положительная и отрицательная. При отрицательном перекосе левый хвост распределения длиннее. При положительном перекосе правый хвост длиннее. Если рассмотреть примеры распределений с конечной асимметрией, то можно выделить следующие варианты. Нормальное распределение и любое другое симметричное распределение с конечным третьим моментом $\left(\mu_{3}\right)$ имеют асимметрию $\gamma_{1}=0$. Например, половина справа нормального распределение имеет

${ }^{36}$ Joanes, D.N.; Gill, C.A. Comparing measures of sample skewness and kurtosis. Journal of the Royal Statistical Society. Series D. 1998. № 47 (1). P. 183-189. DOI: 10.1111/1467-9884.00122. 
асимметрию чуть ниже $\gamma_{1} \leq 1$, а слева $\gamma_{1} \geq-1$. Экспоненциальное распределение имеет асимметрию $\gamma_{1}=2$. Логнормальное распределение может иметь асимметрию любого положительного значения $\gamma_{1}>0$ в зависимости от дисперсии этого распределения ${ }^{37}$.

Четвертым моментом является эксцесс распределения:

$$
\mathbb{M}\left(K-\mu_{1}\right)^{4}=\mu_{4}=\frac{1}{E_{0}} \int_{0}^{+\infty}\left(K-\mu_{1}\right)^{3} \cdot F(K) d K
$$

Эксцессом случайной величины $K$ является третий стандартизированный момент $\gamma_{2}$, его также называют коэффициентом эксцесса, и он определяемый как:

$$
\gamma_{2}=\frac{\mu_{4}}{\sigma^{4}}-3=\frac{\mu_{3}}{\mu_{2}^{2}}-3
$$

«Минус три» в конце формулы введено для того, чтобы коэффициент эксцесса стандартного нормального распределения был равен $\gamma_{2}=0$. Коэффициент эксцесса положителен, если пик распределения около математического ожидания острый, и отрицателен, если пик очень гладкий. Например, острое распределение Лапласа имеет коэффициент эксцесса $\gamma_{2}=3$, а для равномерного распределения $\gamma_{2}=-1.2$. Отсюда единственными наблюдаемыми значениями данных, которые способствуют эксцессу любым значимым способом, являются значения функции распределения вне области пика распределения ${ }^{38}$. Эти значения также являются весьма важными при оценке структурных характеристик неорганических ионообменных материалов.

Стационарный случайный процесс является формой выражения химического равновесия, в связи с этим рассмотрим равновесие ионного обмена. На произвольном элементе $d E$ будет наблюдаться равновесие реакции (1), описываемое уравнением Никольского ${ }^{39}$ :

$$
K=\left(\frac{d E_{M e^{+}}}{d E_{H^{+}}}\right)\left(\frac{a_{H^{+}}}{a_{M e^{+}}}\right)
$$

Причем

$$
d E=d E_{M e^{+}}+d E_{H^{+}}=F(K) d K
$$

${ }^{37}$ Brown S., Oak Road Systems. Measures of Shape: Skewness and Kurtosis. 20082016. URL: https://brownmath.com/stat/shape.htm.

38 Westfall, P.H. Kurtosis as Peakedness, 1905-2014. R.I.P. The American Statistician. 2014. № 68 (3). P. 191-195. DOI: 10.1080/00031305.2014.917055.

39 Кокотов Ю.А, Пасечник В.А. Равновесие и кинетика ионного обмена. Ленинград : Химия, 1970. С. 108. 
Подставляя (28) в (29), получим:

$$
d E_{M e^{+}}+\frac{a_{H^{+}}}{a_{M e^{+}} K} d E_{M e^{+}}=F(K) d K
$$

Отсюда

$$
\frac{d E_{M e^{+}}}{d K}=\frac{F(K)}{\left(1+\frac{a_{H^{+}}}{a_{M e^{+}}}\right)}
$$

Интегрируя (31), получим

$$
E_{M e^{+}}\left(a_{H^{+}}\right)=\int_{0}^{\infty} \frac{F(K)}{\left(1+\frac{a_{H^{+}}}{a_{M e^{+}}}\right)} d K
$$

На практике обычно измеряемыми величинами являются $p H=-\lg \left(a_{H^{+}}\right)$и $p K_{a}=-\lg \left(K_{a}\right)$, поэтому уравнение (32) легко преобразуется к виду:

$$
E_{M e^{+}}(p H)=\ln 10 \int_{-\infty}^{+\infty} \frac{F(p K) 10^{-p K}}{\left(1+10^{p K-p H-\lg a_{M e^{+}}}\right)} d p K
$$

Уравнение (33) представляет собой уравнение кривой потенциометрического титрования или изотермы сорбции в координатах $\mathrm{E}-\mathrm{pH}$. В данном случае мы рассматриваем стандартную модель, которая не учитывает тонкие эффекты, связанные с изменением диэлектрических свойств ионообменного материала и раствора, находящегося в равновесии с ним ${ }^{40}$. Предложенное уравнение изотермы сорбции представляет собой линейное интегральное уравнение Фредгольма 1 рода и может быть с успехом применено к моделированию кривых потенциометрического титрования с различным характером распределения сорбционных центров по константе сорбции.

Проанализируем свойства полученной зависимости. Для удобства рассмотрим уравнение (32). Для щелочной среды, где

$$
\frac{a_{H^{+}}}{a_{M e^{+}}} \ll 1
$$

Отсюда имеем $490 \mathrm{c}$.

${ }^{40}$ Гельферих Ф. Иониты. Москва : Издательство иностранной литературы, 1962. 


$$
E_{M e^{+}}=\int_{n}^{+\infty} F(K) d K=E_{0}
$$

Таким образом, в щелочной среде должно наблюдаться насыщение полной обменной емкости, что реально и наблюдается у большинства гидратированных оксидов. В кислой среде, где

$$
\frac{a_{H^{+}}}{a_{M e^{+}}} \gg 1
$$

Так как

$$
F(K) \ll\left(1+\frac{a_{H^{+}}}{a_{M e^{+}}}\right)
$$

Имеем $E_{M e^{+}}=0$, что также наблюдается у реальных объектов.

Предположим, что распределение центров по константе обмена носит характер $\delta$-функции

$$
F(K)=\sum_{i=1}^{N} \delta\left(K_{i}\right) E_{i}
$$

Отсюда на основании свойств $\delta$-функции становится очевидным следующий вид кривой титрования

$$
E_{M e^{+}}=\sum_{i=1}^{N} \frac{E_{i}}{\left(1+\frac{a_{H^{+}}}{a_{M e^{+}} K_{i}}\right)}
$$

Таким образом, приходим к дискретному описанию равновесия, предложенному в работах ${ }^{41}$. Таким образом, полученное уравнение изотермы обмена является верным, поскольку в пределе при стремлении функции распределения обменных центров ко константе обмена к $\delta$-функции, она стремится к классической изотерме ионного обмена для полифункционального ионообменника.

В завершение этого рассмотрения необходимо подчеркнуть, что уравнения (32), (33), как и (39), обладают свойством зарядовой симметрии. Они справедливы как для протонодонорных, так и для протоноакцепторных центров. Для протоноакцепторных центров уравнение изотермы сорбции аналогично. Это означает что его можно

${ }^{41}$ Белинская Ф.А. Неорганические иониты. Иониты в химической технологии. Ленинград, 1982. С. 158-203; Бобыренко Ю.Я. Распределение кислотных и основных групп поверхности рутила по величине констант диссоциации. Журнал физической химии. 1978. Т. 92. № 56. С. 1488-1490. 
применять как для случаев катионного обмена, так и для анионообменников.

\section{3. Моделирование кривых потенциометрического титрования} при заданной функции распределения обменных центров

Уравнения (32) и (33) представляют собой линейные интегральные уравнения Фредгольма первого рода. В связи с этим эти уравнения могут быть с успехом применены к моделированию кривых потенциометрического титрования по характеру распределения обменных центров по константе обмена. Фактичекски эти уравнения являются уравнением свертки, поскольку свертка - это один из видов интегрального преобразования. Свертка - это математическая операция, примененная к двум функциям, порождающая третью функцию, которую можно рассматривать как модифицированную версию одной из первоначальных. В случае приложения этого преобразования к равновесию ионного обмена мы получаем свертку равновесной кривой, определяемой уравнением закона действующих масс с функцией распределения обменных центров по константе обмена.

Для сравнения возьмем кривой титрования простого двухосновного ионообменного материала, которого функции распределения имеют вид $\delta$-функции $(\sigma=0.0)$, и кривая титрования описывается уравнением (39). Для удобства положим, что $\mathrm{pK}_{1}=5.0, \mathrm{pK}_{2}=10.0$. Рассчитанная для таких условий кривая титрования ионообменного материала примет вид, представленный на рис. 3 .

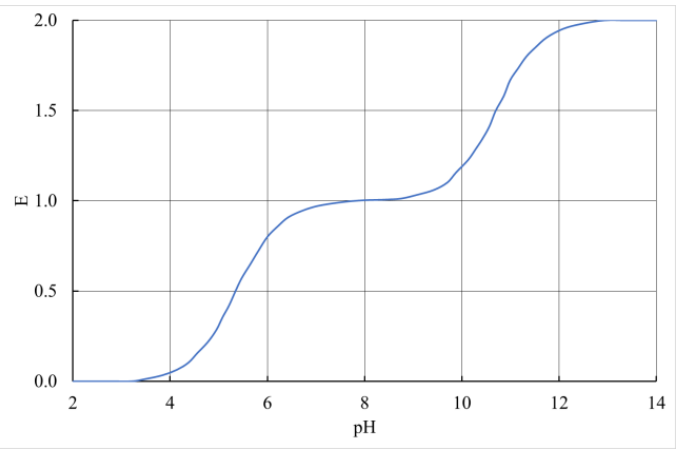

\section{Рис. 3. Расчетная кривая титрования модельного двухосновного ионообменного материала с $\mathrm{pK}_{1}=5.0, \mathrm{pK}_{2}=10.0$. полученная на основе уравнения (39)}

Анализ возможных вариантов поведения кривых титрования при использовании различных вариантов функций распределения обменных центров по константе обмена начнем с нормального 
распределения - функции нормального распределения Гаусса. В нашем случае эта функция распределения будет иметь вид:

$$
F(K)=\frac{1}{\sigma \sqrt{2 \pi}} \exp \left(-\frac{\left(p K-p K_{a v}\right)^{2}}{2 \sigma^{2}}\right)
$$

где $p K_{a v}=-\lg \left(K_{a v}\right)-$ среднее значение отрицательного логарифма константы диссоциации. Для расчетов использовалось уравнение (33).

При этих расчетах концентрация ионов металла принималась постоянной $a_{M e^{+}}=1$. Это означает, что ионная сила раствора постоянна на протяжении всего сорбционного процесса. То есть $a_{H^{+}} \ll a_{M e^{+}}$. Эти условия обычно соответствуют стандартным условиям проведения экспериментов по получению кривых титрования ионообменных материалов. На рис. 4 представлены зависимости, рассчитанные для различных значений дисперсии $\sigma$ обменных центров по константе обмена.

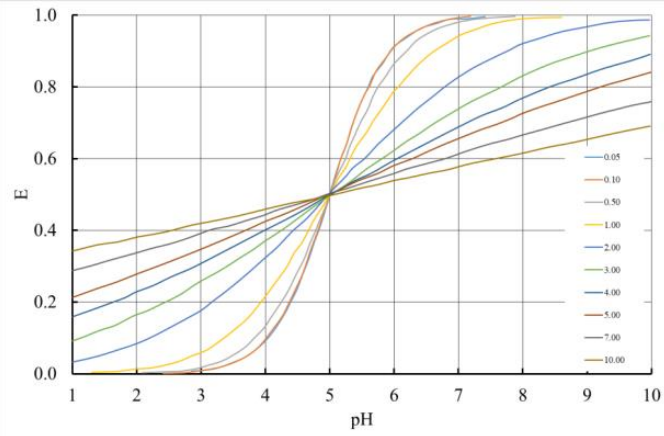

Рис. 4. Кривые титрования полифункционального ионообменного материала, рассчитанные на основе уравнения (33) для распределения обменных центров по константе обмена в соответствии с функцией нормального распределения Гаусса, для различных значений дисперсии (б) этого распределения. Среднее значение $\mathrm{pK}_{\mathrm{av}}=\mathbf{5 . 0}$

Представленные результаты показывают, что при малых значениях дисперсии обменных центров кривые практически не отличаются от аналогичных зависимостей для монофункционального ионообменника. С ростом дисперсии кривые титрования становятся более пологими, точка перегиба при этом остается на одном месте. Представляло интерес проанализировать, как меняется первая производная емкости ионообменника по рН в точке перегиба в зависимости от дисперсии: 


$$
\left(\frac{d E}{d p H}\right)_{p K_{a v}=5.0}=f(\sigma)
$$

Поскольку в точке перегиба $p K_{a v}=p H_{E=0.5}=5.0, \quad$ то вполне логичным является использование величины $\left(\frac{d E}{d p H}\right)_{p H=5.0}$ в качестве индивидуальной характеристики изменения кривой титрования в зависимости от дисперсии обменных центров по константе обмена. Полученные результаты представлены на рис. 5.

Из полученной зависимости хорошо видно, что с уменьшением дисперсии и с приближением функции нормального распределения константы обмена к $\delta$-функции производная в точке перегиба стремится к некоторой постоянной величине. Однако с ростом дисперсии первая производная от кривой титрования по $p H$ в точке перегиба монотонно уменьшается.

В качестве следующего модельного варианта при расчетах кривой титрования выберем функцию полунормального распределения:

$$
F(p K, \sigma)=\left\{\begin{array}{c}
\frac{\sqrt{2}}{\sigma \sqrt{\pi}} \exp \left(-\frac{\left(p K-p K_{\text {edge }}\right)^{2}}{2 \sigma^{2}}\right) p K \geq p K_{\text {edge }} \\
0 p K<p K_{\text {edge }}
\end{array}\right.
$$

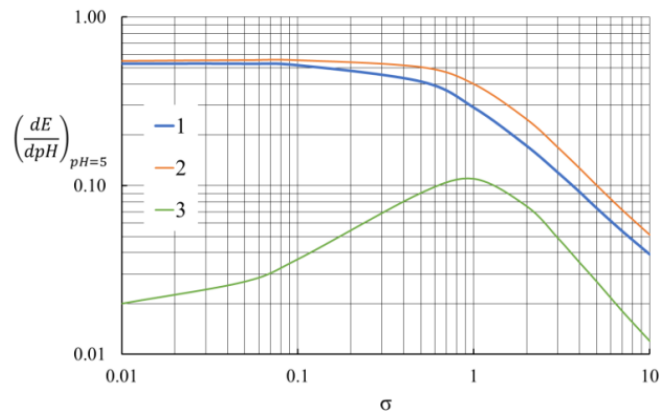

Рис. 5. Изменение значения первой производной от кривой титрования по $p H$ в точке перегиба $\left(\frac{d E}{d p H}\right)_{p H=5.0}$ для различных функций распределения по константе обмена в зависимости от дисперсии распределения $\sigma .1$ - Функция нормального распределения Гаусса (GD). 2 - Функция прямоугольного равномерного распределения (CUD).

$$
3-\text { разность } \Delta=\left(\frac{d E}{d p H}\right)_{p H=5.0}^{G D}-\left(\frac{d E}{d p H}\right)_{p H=5.0}^{C U D}
$$


В теории вероятностей и статистике полунормальное распределение является частным случаем сложенного нормального распределения, а именно сложенного по середине обычного нормального распределения с нулевым средним. В нашем случае сложение производим в точке, соответствующей максимуму функции нормального распределения $-p K_{\text {edge }}$. Сложение проводим в правую сторону. Кривые титрования полифункционального ионообменного материала, рассчитанная на основе уравнения (33) для распределения обменных центров по константе обмена в соответствии с половинной справа функцией нормального распределения (42), для различных значений дисперсии (б) этого распределения представлены на рис. 6 .

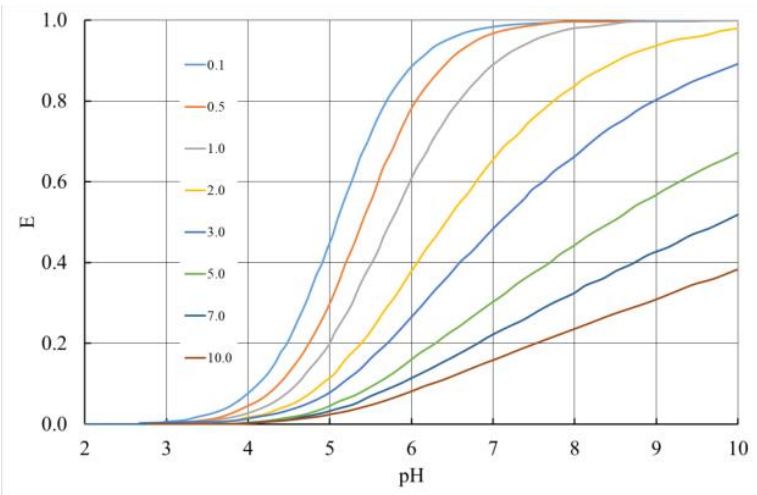

Рис. 6. Кривые титрования полифункционального ионообменного материала, рассчитанная на основе уравнения (33) для распределения обменных центров по константе обмена в соответствии с половинной справа функцией нормального распределения, для различных значений дисперсии $(\sigma)$ этого распределения. Крайнее левое значения $\mathrm{pK}_{\text {edge }}=5.0$

Из полученных зависимостей хорошо видно, что с увеличением дисперсии распределения обменных центров по константе обмена наблюдается значительное растягивание кривой титрования в сторону больших значений $\mathrm{pH}$. Проанализируем изменение величины $\mathrm{pH}_{(E=0.5)}$, соответствующей половинному заполнению обменных центров ионообменника. Полученные расчетные данные представлены на рис. 7. 


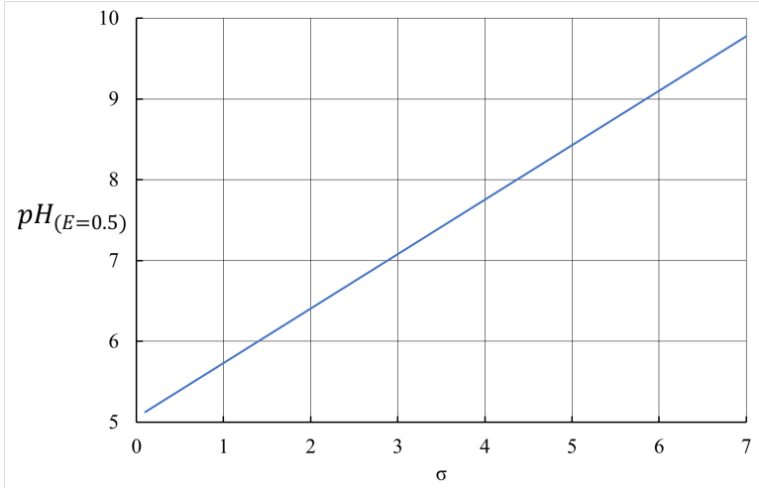

Рис. 7. Смещение точки, соответствующей половинному заполнению обменных центров ионообменника $\left(\mathrm{pH}_{E=0.5}\right)$, в зависимости о дисперсии половинной справа функцией нормального распределения. Крайнее левое значение $p K_{\text {edge }}=5.0$

Из данных, представленных на рис. 7, видно, что $p H_{E=0.5}$ точки, соответствующей половинному заполнению обменных центров ионообменника, линейно смещается в сторону больших значений $\mathrm{pH}$. Производная от этой зависимости по $\sigma$ равна:

$$
\frac{d p H_{E=0.5}}{d \sigma}=0.674
$$

Однако из свойств полунормального распределения известно, что его среднее имеет вид:

$$
\mathbb{M} K=\mu_{1}=\frac{\sigma \sqrt{2}}{\sqrt{\pi}}=0.797884561 \cdot \sigma
$$

Отсюда следует, что сдвиг точки $p H_{E=0.5}$ происходит медленнее, чем это происходит со средним значением функции полунормального распределения. Этот эффект можно связать с наличием влияния самого интегрального предобразования, которое происходит при применении уравнения (33). Обнаруженный факт еще раз подтверждает то, что нельзя простым дифференцированием кривой титрования получить функцию распределения обменных центров по константе обмена.

В качестве следующего модельного варианта при расчетах кривой титрования выберем другой вид функции полунормального распределения, а именно функцию, сложенную в левую сторону: 


$$
F(p K, \sigma)=\left\{\begin{array}{c}
\frac{\sqrt{2}}{\sigma \sqrt{\pi}} \exp \left(-\frac{\left(p K-p K_{\text {edge }}\right)^{2}}{2 \sigma^{2}}\right) p K \leq p K_{\text {edge }} \\
0 p K>p K_{\text {edge }}
\end{array}\right.
$$

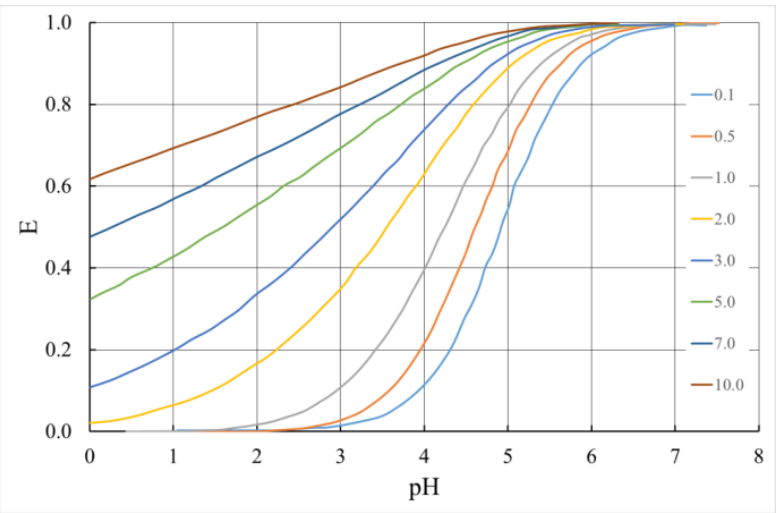

Рис. 8. Кривые титрования полифункционального ионообменного материала, рассчитанная на основе уравнения (33) для распределения обменных центров по константе обмена в соответствии с половинной слева функцией нормального распределения, для различных значений дисперсии ( $\sigma)$ этого распределения. Крайнее правое значения $\mathrm{pK}_{\mathrm{edge}}=5.0$

Кривые титрования полифункционального ионообменного материала, рассчитанные для этого вида функции распределения, представлены на рис. 8 .

Из полученных зависимостей хорошо видно, что с увеличением дисперсии распределения обменных центров по константе обмена наблюдается значительное растягивание кривой титрования в сторону меньших значений $\mathrm{pH}$. Проанализируем изменение величины $p H_{(E=0.5)}$, соответствующей половинному заполнению обменных центров ионообменника. Полученные расчетные данные представлены на рис. 9.

Из данных, представленных на рис. 9, видно, что значения $p H_{E=0.5}$ точки, соответствующей половинному заполнению обменных центров ионообменника, линейно смещается в сторону меньших значений $\mathrm{pH}$. Производная от этой зависимости по $\sigma$ ведет себя аналогично предыдущему случаю, только меняет знак на противоположный.

В качестве следующего модельного варианта при расчетах кривой титрования также выберем функцию сложенного нормального распределения. В отличие от предыдущего случая, возьмем вариант 
распределения, соответствующий уравнению (46). Данный вариант распределения представляет собой нормальное распределение, ограниченное симметрично с двух сторон относительно центра этого распределения.

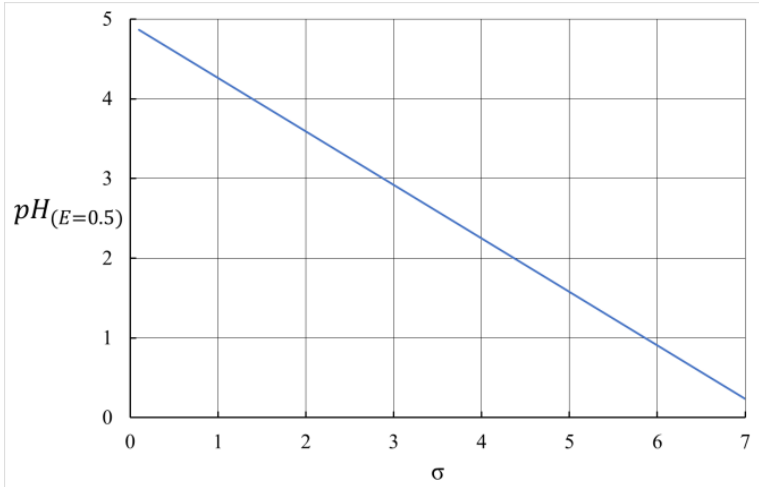

Рис. 9. Смещение точки, соответствующей половинному заполнению обменных центров ионообменника $\left(\mathrm{pH}_{E=0.5}\right)$, в зависимости от дисперсии половинной слева функцией нормального распределения. Крайнее правое значение $p K_{\text {edge }}=5.0$

$$
\begin{aligned}
& F\left(p K, \sigma, p K_{\text {min }}, p K_{\max }\right) \\
& =\left\{\begin{array}{c}
\exp \left(-\frac{\left(p K-p K K_{a v}+p K_{\min }\right)^{2}}{2 \sigma^{2}}\right) \\
\frac{1}{\sigma 2 \sqrt{2 \pi}}\left[\begin{array}{c}
\exp \left(-\frac{\left(p K+p K_{a v}-p K_{\min }\right)^{2}}{2 \sigma^{2}}\right) \\
+\exp \left(-\frac{\left(p K-p K_{a v}+p K_{\max }\right)^{2}}{2 \sigma^{2}}\right) \\
+\exp \left(-\frac{\left(p K+p K_{a v}-p K_{\max }\right)^{2}}{2 \sigma^{2}}\right) \\
0, p K>p K_{\max }
\end{array}\right], p K_{\min } \leq p K \leq p K_{\max }
\end{array}\right.
\end{aligned}
$$

Рассчитанные кривые титрования полифункционального ионообменного материала, рассчитанные на основе уравнения (33) для распределения обменных центров по константе обмена в соответствии с функцией нормального распределения ограниченной с двух сторон, для различных значений дисперсии $(\sigma)$ этого распределения, 
представлены на рис. 10. $p K_{\min }$ и $p K_{\max }-$ границы этого распределения.

Из полученных зависимостей хорошо видно, что с увеличением дисперсии распределения обменных центров по константе обмена наблюдается их сложное изменение, причем эти изменения в значительной мере наблюдаются при больших значениях рН, и кривые титрования растягиваются в сторону больших значений $\mathrm{pH}$.

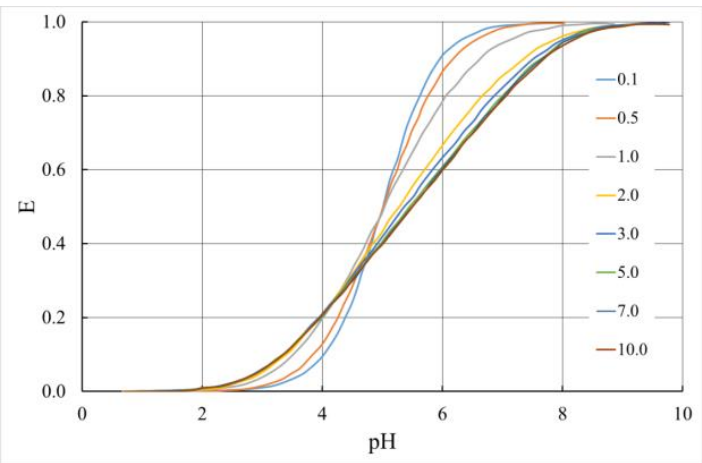

Рис. 10. Кривые титрования полифункционального ионообменного материала, рассчитанные на основе уравнения (33) для распределения обменных центров по константе обмена в соответствии с функцией распределения Гаусса, ограниченной с двух сторон (46), для различных значений дисперсии (б) этого распределения. Среднее значение $\mathrm{pK}_{\mathrm{av}}=\mathbf{5 . 0}$. Границы распределения соответствуют: $p K_{\min }=3.0, p K_{\max }=7.0$

По аналогии с предыдущими случаями проанализируем изменение величины $p H_{(E=0.5)}$, соответствующей половинному заполнению обменных центров ионообменника. Полученные расчетные данные представлены на рис. 11.

Из представленной зависимости видно, что при малых значениях дисперсии обменных центров по константе обмена кривые титрования ведут себя, как и в случае нормального распределения. То есть вклад в суммарный результат от крыльев распределения за пределами границ распределения незначителен. Однако когда их вклад с ростом дисперсии распределения растет, начинается существенный сдвиг значения $p H_{E=0.5}$ в сторону больших значений $\mathrm{pH}$. Причем этот сдвиг вначале происходит быстро, а затем асимптотически стремится к некоторому фиксированному значению. В пределе увеличения дисперсии эта функция распределения стремится к другому виду 
функции распределения, а именно к непрерывному равномерному распределению.

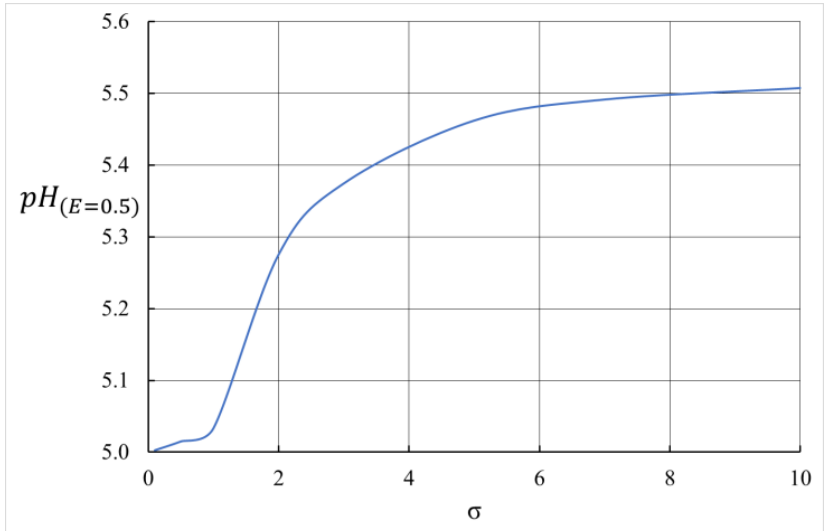

Рис. 11. Смещение точки, соответствующей половинному заполнению обменных центров ионообменника $\left(p H_{E=0.5}\right)$,

в зависимости от дисперсии функции распределения Гаусса, ограниченной с двух сторон. Среднее значение $\mathrm{pK}_{\mathrm{av}}=5.0$.

Границы распределения соответствуют: $p K_{\min }=3.0, p K_{\text {max }}=7.0$

В связи с этим нами была предпринята попытка моделирования кривых титрования с использованием этого варианта функции распределения обменных центров по константе обмена.

Непрерывное равномерное распределение в теории вероятностей, распределение случайной вещественной величины, принимающей значения, принадлежащие интервалу $\left[p K_{\min }, p K_{\max }\right]$, характеризующееся тем, что плотность вероятности на этом интервале постоянна. В принятом для расчета случае функция непрерывного равномерного распределения имеет вид, представленный уравнением (47):

$$
F\left(p K, p K_{\min }, p K_{\max }\right)=\left\{\begin{array}{c}
\frac{1}{p K_{\max }-p K_{\min }}, p K \in\left[p K_{\min }, p K_{\max }\right] \\
0, p K \notin\left[p K_{\min }, p K_{\max }\right]
\end{array}\right.
$$

Дисперсия для данного распределения описывается уравнением (48):

$$
\sigma=\sqrt{\frac{\left(p K_{\max }-p K_{\min }\right)^{2}}{12}}=\frac{p K_{\max }-p K_{\min }}{2 \sqrt{3}}
$$


Отсюда можно легко получить значения границ этого распределения при заданных значениях среднего $\left(p K_{a v}\right)$ и дисперсии:

$$
\begin{aligned}
& p K_{\text {min }}=p K_{a v}-\sigma \sqrt{3} \\
& p K_{\text {max }}=p K_{a v}+\sigma \sqrt{3}
\end{aligned}
$$

Рассчитанные кривые титрования полифункционального ионообменного материала, рассчитанные на основе уравнения (33) для распределения обменных центров по константе обмена в соответствии с функцией непрерывного равномерного распределения (47), полученные с учетом выражений (49) и (50), представлены на рис. 12.

Как и для функций нормального распределения, в данном случае логичным является использование функции (35), отражающей изменение величины первой производной от кривой титрования по $\mathrm{pH}$ в точке перегиба $\left(\frac{d E}{d p H}\right)_{p H=5.0}$, в зависимости от дисперсии обменных центров по константе обмена, в качестве индивидуальной характеристики изменения кривой титрования. Полученные результаты представлены на рис. 5 .

Из полученных результатов видно, что зависимость (35) ведет себя аналогично подобной зависимости для функции нормального распределения. Однако у нее есть некоторая особенность, отличающая ее от варианта с функцией нормального распределения. Характер этой особенности проявляется при анализе разности этих зависимостей:

$$
\Delta=\left(\frac{d E}{d p H}\right)_{p H=5.0}^{G D}-\left(\frac{d E}{d p H}\right)_{p H=5.0}^{C U D}
$$




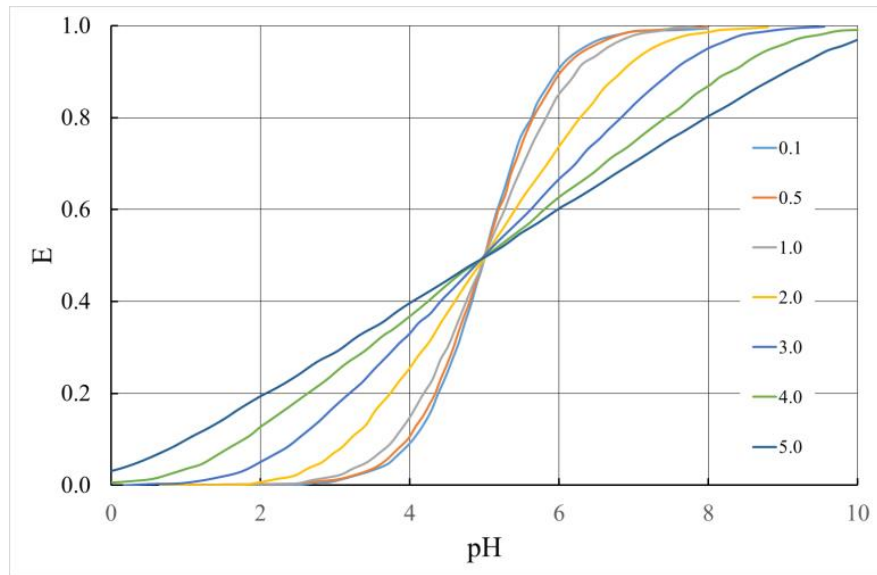

Рис. 12. Кривые титрования полифункционального ионообменного материала, рассчитанные на основе уравнения (33) для распределения обменных центров по константе обмена в соответствии с функцией равномерного непрерывного прямоугольного распределения, для различных значений дисперсии (б) этого распределения. Среднее значение $\mathrm{pK}_{\mathrm{av}}=5.0$

Здесь индекс GD относится к варианту, рассчитанному на основе функции нормального распределения, а индекс CUD - к варианту на основе функции равномерного непрерывного прямоугольного распределения. Из кривой 3 , изображенной на рис. 5 , видно, что при малых и больших значениях дисперсии эти зависимости мало отличаются друг от друга по своему поведению. При малых значениях дисперсии они обе стремятся к $\delta$-функции распределения. При больших значениях дисперсии функция равномерного непрерывного прямоугольного распределения стремится к виду, близкому к функции нормального распределения, в виду их значительного уширения. Однако при средних значениях диспепсии их различия становятся существенными.

Таким образом, опираясь на проведенный анализ, можно увидеть, что получаемые расчетные кривые титрования имеют часто похожий вид с экспериментально полученными кривыми. Это указывает на то, что, исходя из внешнего вида полученной экспериментально кривой титрования соответствующего ионообменного материала, можно сделать предварительную качественную оценку вида функции распределения обменных центров по константе обмена. Эта оценка в дальнейшем облегчит задачу расчета функции распределения исходя из полученных экспериментальных данных. 


\section{4. Пути решения обратной задачи восстановления функции распределения по кривым титрования}

Помимо моделирования кривых титрования, часто необходимо решать обратную задачу: восстановление функции распределения по кривой титрования. Эта задача является некорректно поставленной по Адамару ${ }^{42}$, т. е. сколь угодно малые изменения наблюдаемой функции могут соответствовать сколь угодно большим изменениям определяемой зависимости ${ }^{43}$. Природа этого факта состоит в том, что интегральные операторы в правых частях уравнений (32), (33) являются сглаживающими, поэтому при решении обратной задачи, чтобы объяснить малые возмущения в кривой титрования, приходится предполагать значительные отклонения функции распределения $F(K)$.

Рассмотрим возможность аналитического решения уравнения (32). Само уравнение (33) относится к интегральным уравнениям I рода с постоянными пределами интегрирования. Искомой функцией является функция распределения по константам обмена $\mathrm{F}(\mathrm{K})$. Функция $E_{M e^{+}}\left(a_{H^{+}}\right)$является экспериментальной измеряемой функцией. Функция $\Phi\left(a_{H^{+}}, K\right)$ является ядром интегрального уравнения (32):

$$
\Phi\left(a_{H^{+}}, K\right)=\frac{1}{\left(1+\frac{a_{H^{+}}}{a_{M e^{+}}}\right)}=\frac{a_{M e^{+}}}{\left(a_{M e^{+}} K+a_{H^{+}}\right)}=\frac{K}{\left(K+\frac{a_{H^{+}}}{a_{M e^{+}}}\right)}
$$

Главным условием возможности решения интегрального уравнения является то, что ядро должно быть представлено непрерывной функцией во всех точках области определения. В нашем случае функция (52) является очевидно непрерывной во всем диапазоне возможных значений аргументов $0<K<\infty$ и $0 \leq a_{H^{+}}<\infty$. Анализ литературных данных по методам решения интегральных уравнений Фредгольма I-го рода показал, что отсутствуют варианты строгого аналитического решения этой задачи ${ }^{44}$.

Помимо этого, следует особо подчеркнуть, что сведение задачи только к решению интегрального уравнения существенно идеализирует

42 Тихонов А.Н., Арсенин В.Я. Методы решения некорректных задач. Москва : Наука, 1974.

${ }_{43}$ Иванов В.К., Васин В.В., Танана В.П. Теория линейных некорректных задач и ее приложения. Москва : Наука, 1978.

${ }^{44}$ Каток А.Б., Хассельблат Б. Введение в современную теорию динамических систем с обзором последних достижений / пер. с англ. под ред. А.С. Городецкого. Москва : МЦНМО, 2005. 464 с.; Тихонов А.Н., Арсенин В.Я. Методы решения некорректных задач. Москва : Наука, 1974; Иванов В.К., Васин В.В., Танана В.П. Теория линейных некорректных задач и ее приложения. Москва : Наука, 1978; Вапник В.Н., Глазкова Т.Г., Кощеев В.А., Михальский А.И., Червоненкис А.Я. Алгоритмы и программы восстановления зависимостей. Москва : Физматлит, 1984. 816 с.; Полянин А.Д., Манжиров А.В. Справочник по интегральным уравнениям. Москва : Физматлит, 2003. 
ситуацию, поскольку наблюдения производится лишь на конечном множестве значений $\mathrm{pH}$ или концентраций и, как результат, мы все равно не имеем точного аналитического описания функции, стоящей в левой части уравнений (32) и (33). Максимум, что можно получить из экспериментальных данных, это аппроксимацию этой фикции одним из стандартных методов. Таким образом, опираясь только на условия (17) - (20), задача становится неразрешимой. Однако ее можно решить, если предположить, что решение принадлежит достаточному узкому классу функций или может быть хорошо приближено функцией такого класса. В первом случае решение будет зависеть от конечного числа параметров и может быть найдено методом наименьших квадратов, если количество наблюдений превышает число параметров ${ }^{45}$. Во втором случае решение может быть найдено путем введения регуляризирующего функционала ${ }^{46}$. На практике удобнее всего использовать конечное разложение по полиномам Чебышева или сплайны с конечным числом сопряжений, например, кубические. Таким образом, по данным потенциометрического титрования полимерных оксигидратов металлов, полученных золь-гель методами, можно получать данные о распределении активных протонодонорных и акцепторных центров по константе сорбции.

В литературе описаны методы решения интегральных уравнений Фредгольма I-го рода, которые строятся в классе кусочно-постоянных функций ${ }^{47}$. В основе этих методов лежит условие равенства нулю средних значений «невязок» уравнений на каждом интервале из множества разбиений области построения решения. Число интервалов $\mathrm{N}$ и их распределение при заданном числе определяются из условия минимума среднеквадратичного отклонения по всей области «невязки». Решения строятся для последовательности значений $\mathrm{N}$, приближенные распределения определяются численно, например, методом покоординатного спуска.

Рассмотри предлагаемую процедуру. Упростим уравнение (32) с учетом (52) и получим следующее уравнение в общем виде:

${ }^{45}$ Вапник В.Н., Глазкова Т.Г., Кощеев В.А., Михальский А.И., Червоненкис А.Я. Алгоритмы и программы восстановления зависимостей. Москва : Физматлит, 1984. $816 \mathrm{c}$.

46 Тихонов А.Н., Арсенин В.Я. Методы решения некорректных задач. Москва : Наука, 1974.

Латыпов А.Ф. Численные методы решения линейных интегральных уравнений Фредгольма и Вольтерра 1-го рода. Сборник докладов Международной конференции "Обратные $и$ некорректные задачи математической физики», посвященная 75-летию академика М.М. Лаврентьева, 20-25 августа 2007 г., Новосибирск. 


$$
\int_{0}^{\infty} \frac{t y(t) d t}{t+a x}=f(x)
$$

Ядро этого уравнения имеет вид:

$$
K(x, t)=\frac{t}{t+a x}
$$

Правая часть $f(x)$ - экспериментально определенная интегрируемая функция. Пределы интегрирования заменим на конечный диапазон экспериментально значимых значений констант диссоциации, которым соответствует параметр $t \in[a, b]$. Диапазон изменения аргумента $x$ также будем рассматривать на конечном диапазон экспериментально значимых значений $x \in[c, d]$. Разобьем отрезок $[a, b]$ на $n$ интервалов, $t_{j}$ - точки разбиения, номера интервалов, $j=1 \div n+1, t_{1}=a, t_{n+1}=b$. Пусть на $j$-ом интервале $y(t)=y_{j}=$ const и обозначим

$$
\int_{t_{j}}^{t_{j+1}} K(x, t) d t=k_{j}\left(x, t_{j}, t_{j+1}\right)
$$

Тогда уравнение (53) с учетом (55) запишется в следующем виде

$$
z(x)=\sum_{j=1}^{n} k_{j}\left(x, t_{j}, t_{j+1}\right) y_{j}-f(x)=0
$$

Разобьем отрезок $[c, d]$ также на $n$ интервалов; $x_{i}$ - точки разбиения, соответствующие экспериментальным значениям аргумента или узлам аппроксимирующей функции; номера интервалов $i=1 \div n+1, x_{1}=c$, $x_{n+1}=d$. Примем, что на каждом $i$-ом интервале уравнения (56) удовлетворяют решению в «среднем», т.е.

$$
\langle z(x)\rangle=\frac{1}{\Delta x_{i}} \int_{x_{i}}^{x_{i+1}} z(x) d x=0
$$

Введем следующие обозначения

$$
\left\langle k_{j}\left(x, t_{i}, t_{i+1}\right)\right\rangle=\frac{1}{\Delta x_{i}} \int_{x_{i}}^{x_{i+1}} k_{j}\left(x, t_{i}, t_{i+1}\right) d x=k_{i j}\left(t_{i}, t_{i+1}\right)
$$




$$
\langle f(x)\rangle=\frac{1}{\Delta x_{i}} \int_{x_{i}}^{x_{i+1}} f(x) d x=f_{i}
$$

Здесь $f_{i}$ - экспериментально измеренные значения функции или значения аппроксимирующей функции в узловых точках. Полученные величины в уравнении (58) представляют собой матрицу:

$$
M=\left\{k_{i j}\left(t_{i}, t_{i+1}\right)\right\}
$$

Значения $y_{j}$ и $f_{i}$ представляют собой матрицы-столбцы:

$$
\begin{gathered}
Y=\left\{y_{j}\right\} \\
F=\left\{f_{i}\right\}
\end{gathered}
$$

Отсюда из уравнения (56) с учетом выражений (60) - (62) получим уравнение в матричной форме:

$$
M \times Y=F
$$

Решение уравнения (63) будет иметь вид:

$$
Y=M^{-1} \times F
$$

Результат вычисления решения по уравнению (64) будет зависеть от числа разбиений $n$ и распределения точек при разбиении интервалов $\left\{x_{i}\right\}$ и $\left\{t_{i}\right\}$.

Для поиска оптимального решения введем функционал:

$$
\Phi\left(n, x_{i}, t_{j}\right)=\int_{c}^{d} z^{2}(x) d x
$$

Отсюда получим искомое решение:

$$
\Phi^{*}=\min _{n, x_{i}, t_{j}} \Phi\left(n, x_{i}, t_{j}\right), x_{i-1}<x_{i}<x_{i+1}, t_{j-1}<t_{j}<t_{j+1} ; i, j=2 \div n
$$

Вычисления по уравнению (66) дают искомое решение. Задача (66) решается приближенно, так как получение достаточно точного решения требует большого объема вычислений. Кроме того, при проведении вычислений может оказаться недостаточным разбиения интервалов только на экспериментальные точки. С большой 
вероятностью можно сказать, что потребуется аппроксимация экспериментальных зависимостей определенными функциями или сплайнами для увеличения количества точек на выбранных интервалах. Большие искажения в получаемые результаты может внести и разброс экспериментальных точек. Поэтому аппроксимация может иметь и другую важную функцию, такую как сглаживание экспериментальных зависимостей. При этом также важно учитывать правильный подбор аппроксимирующих функций, так как неправильная аппроксимация может привести к искажению первичных данных и получению конечных результатов весьма далеких от истины.

Функционал среднеквадратичной «невязки» представляет собой функцию, имеющую несколько локальных экстремумов. Это обстоятельство необходимо учитывать при выборе метода решения задачи (66). Для подобных систем обычно рекомендуется используется алгоритм покоординатного сканирования функционала на заданном числе точек с выбором лучшей точки для последовательности значений $\boldsymbol{n}$. В общем случае алгоритм не позволяет получить глобальный минимум функционала, поскольку для этого требуется громадный объем вычислений. Однако по сравнению с алгоритмами поиска локального экстремума он дает существенно меньшие значения функционала. Кроме того, после каждого цикла итераций или группы итераций рекомендуется проверять получаемый результат на соответствие физическому смыслу.

\section{ВЫВОДЫ}

В настоящей работе проведен анализ подходов к описанию неоднородности обменных центров ионообменных материалов. Показаны причины возникновения неоднородности этих центров. Выдвинута и обоснована идея статистического подхода к описанию ионообменного равновесия на неорганических ионообменных материалах. Предложена методика описания ионообменных равновесий с использованием методов статистической термодинамики.

На основе разработанного подхода и в рамках модели полифункционального ионита было предложено уравнение изотермы ионного обмена однозарядных ионов, описывающее обмен на ионите, характеризующимся определенной функцией распределения обменных центров по константе обмена. Предложены пути построения функции распределения обменных центров по данным потенциометрического титрования. Предложенное уравнение изотермы сорбции представляет собой линейное интегральное уравнение Фредгольма первого рода и может быть с успехом применено к моделированию кривых потенциометрического титрования с различным характером распределения сорбционных центров по константе сорбции. 
В соответствии с предложенным уравнением изотермы ионного обмена проведено моделирование кривых потенциометрического титрования при заданной функции распределения обменных центров по константе обмена. Опираясь на проведенный анализ, показано, что получаемые расчетные кривые титрования имеют часто похожий вид с экспериментально наблюдаемыми зависимостями. Это указывает на то, что, исходя из внешнего вида полученной экспериментально кривой титрования соответствующего ионообменного материала, можно сделать предварительную качественную оценку вида функции распределения обменных центров по константе обмена. Эта оценка в дальнейшем облегчит задачу расчета функции распределения исходя из полученных экспериментальных данных.

На основе проведенного анализа предложены пути решения обратной задачи восстановления функции распределения по кривым титрования. Данная задача является некорректно поставленной по Адамару, т. е. сколь угодно малые изменения наблюдаемой функции могут соответствовать сколь угодно большим изменениям определяемой зависимости. Анализ литературных данных по методам решения интегральных уравнений Фредгольма Первого рода показал, что отсутствуют варианты строгого аналитического решения этой задачи. Для численного решения этой задачи предложен вариант метода решения интегрального уравнений Фредгольма Первого рода, который строятся в классе кусочно-постоянных функций. Для минимизации предложенного функционала рекомендовано использование алгоритма его покоординатного сканирования на заданном числе точек с выбором лучшей точки для последовательности значений.

Таким образом, по данным потенциометрического титрования полимерных оксигидратов металлов, полученных различными методами, можно получать данные о распределении активных протонодонорных и акцепторных центров по константе сорбции.

В завершение этого рассмотрения необходимо подчеркнуть, что полученные уравнения обладают свойством зарядовой симметрии. Они справедливы как для протонодонорных, так и для протоноакцепторных центров. Для протоноакцепторных центров уравнение изотермы сорбции аналогично. Это означает что его можно применять как для случаев катионного обмена, так и для анионообменников.

\section{АННОТАЦИЯ}

В рамках модели полифункционального ионита предложено уравнение изотермы ионного обмена однозарядных ионов, описывающее обмен на ионите, характеризующимся определенной функцией распределения обменных центров по константе обмена. Предложены пути построения функции распределения обменных 
центров по данным потенциометрического титрования. Для протонодонорных центров изотерма сорбции имеет вид:

$$
E_{M e^{+}}=\int_{0}^{\infty} \frac{F(K)}{\left(1+\frac{a_{H^{+}}}{a_{M e^{+}}}\right)} d K
$$

где: $E_{M e^{+}}$- экспериментально определяемая степень насыщения активных центров; $F(K)$ - функция распределении активных центров по константе ионного обмена; $a_{H^{+}}$и $a_{M e^{+}}-$активности соответствующих ионов $\mathrm{H}^{+}$и $\mathrm{Me}^{+} ; K-$ константа ионного обмена. Для протоноакцепторных центров уравнение изотермы сорбции аналогично.

\section{ЛИТЕРАТУРА}

1. Кокотов Ю.А, Пасечник В.А. Равновесие и кинетика ионного обмена. Ленинград : Химия, 1970. С. 108.

2. Белинская Ф.А. Неорганические иониты. Иониты в химической технологии. Ленинград, 1982. С. 158-203.

3. Ласкорин Б.Н., Стрелко В.В., Стражеско Д.Н., Денисов В.И. Сорбенты на основе силикагеля в радиохимии. Химические свойства. Применение. Москва : Атомиздат, 1977. 304 с.

4. Вольхин В.В., Онорин С.А. Сорбционные свойства гидратированной двуокиси титана и продуктов ее обезвоживания. Известия АН СССР. Неорганические материаль. 1976. Т. 12. № 8. C. $1415-1418$.

5. Онорин С.А., Вольхин В.В., Змльберман М.В., Ходяшев Н.Б. Синтез катионитов с повышенной обменной емкостью. Известия $A H$ СССР. Неорганические материаль. 1978. Т. 14. С. 150-153.

6. Кудрявцев П.Г., Онорин С.А., Вольхин В.В. Неорганический ионообменник ИСН-1, селективный к ионам лития. Изв. ВУЗ. Цветная металлургия. 1977. № 3. С. 50-53.

7. Белинская Ф.А. Ступенчатое ионообменное равновесие. Модельные представления. Вестник ЛГУ. 1983. № 22. С. 42-48.

8. Бобыренко Ю.Я. Распределение кислотных и основных групп поверхности рутила по величине констант диссоциации. Журнал физической химии. 1978. Т. 92. № 56. С. 1488-1490.

9. Бобыренко Ю.Я. О потенциометрическом методе определения электроповерхностных свойств дисперсных материалов. Коллоидный журнал. Т. 48. № 4. С. 649-653.

10. Рогинский С.3. Гетерогенный катализ. Некоторые вопросы теории. Москва : Наука, 1979. 
11. Кудрявцев П.Г. Равновесие ионного обмена на оксигидратных сорбентах, осложненное дисперсией обменных центров по константе обмена. Журнал физ. Химии. 1987. Т. 61. № 3. С. 848-851.

12. Никольский Б.П., Парамонова В.И. Законы обмена ионов между твердой фазой и раствором. Усn. хим., 1939. Т. 8. С. 1535-1537.

13. Никольский Б.П. О классификации ионитов в свете современной теории обмена ионов. Хроматография. Ленинград, 1956. С. 5-15.

14. Гельферих Ф. Иониты. Москва : Издательство иностранной литературы, 1962. 490 с.

15. Ионный обмен / под ред. Я. Маринского. Москва : Мир, 1968. $565 \mathrm{c}$.

16. Самсонов Г.В. Термодинамические, кинетические и динамические особенности ионного обмена с участием ионов органических веществ. Ионный обмен. Ленинград, 1981. С. 126-137.

17. Челищев Н.Ф. Ионообменные свойства минералов / АН СССР, Институт минералогии, геохимии и кристаллохимии редких элементов. Москва : Наука, 1973. 203 с.

18. Гриссбах Р. Теория и практика ионного обмена. Москва : Издательство иностранной литературы, 1963, 499 с.

19. Белинская Ф.А., Милицина Э.А. Неорганические ионообменные материалы на основе труднорастворимых соединений сурьмы (V). Успехи химии. 1980. Т. 49. № 10. С. 1904-1936.

20. Брек Д. Цеолитовые молекулярные сита. Ленинград, 1976. 781 с.

21. Кудрявцев П.Г., Онорин С.А. Сорбция ионов щелочных металлов гидратированной пятиокисью ниобия. Неорганические ионообменники (синтез, структура, свойства). Пермь, 1977. № 212. C. $105-108$.

22. Mortimer C.T. Reactions heats and bonds strengths. Oxford : Pergamon Prass, 1962.

23. Кудрявцев П.Г., Онорин С.А., Вольхин В.В. Влияние условий осаждения на строение и ионообменные свойства гидратированного оксида алюминия. Известия ВУЗ. Цветная металлургия. 1990. № 2. С. 21-26.

24. Кудрявцев П.Г., Онорин С.А., Вольхин В.В. Влияние термообработки на ионообменные свойства и строение материалов, содержащих гидратированный оксид алюминия. Известия ВУЗ. Цветная металлургия. 1990. № 3. С. 26-31.

25. Кудрявцев П.Г., Онорин С.А., Вольхин В.В. Структурообразование и ионообменные свойства смешанных оксидов в системе $\mathrm{Li}_{2} \mathrm{O}-\mathrm{Fe}_{2} \mathrm{O}_{3}-\mathrm{Nb}_{2} \mathrm{O}_{5}$. Известия AH CССР. Сер. Неорганические материалы. 1991. Т. 27. № 7. С. 1479-1482.

26. Кудрявцев П.Г., Онорин С.А., Вольхин В.В., Ходяшев Н.Б., Ходяшев М.Б., Вольхин Д.В. Неорганические сорбенты на основе гидроксидов металлов для извлечения микропримесей из жидких сред. 
Химия, технология, промышленная экология неорганических соединений. 1998. Вып. 1. С. 101-106.

27.Никольский Б.П. (ред.) Физическая химия. Теоретическое и практическое руководство. Ленинград : Химия, 1987. 880 с.

28. Каток А.Б., Хассельблат Б. Введение в современную теорию динамических систем с обзором последних достижений / пер. с англ. под ред. А.С. Городецкого. Москва : МЦНМО, 2005. 464 с.

29. Spanos A. Probability theory and statistical inference. Econometric modeling with observational data. New York : Cambridge University Press, 1999. $815 \mathrm{p}$.

30. Joanes, D.N., Gill, C.A. Comparing measures of sample skewness and kurtosis. Journal of the Royal Statistical Society. Series D. 1998. № 47 (1). P. 183-189. DOI: 10.1111/1467-9884.00122.

31. Brown S., Oak Road Systems. Measures of Shape: Skewness and Kurtosis, 2008-2016. URL: https://brownmath.com/stat/shape.htm.

32. Westfall, P.H. Kurtosis as Peakedness. 1905-2014. R.I.P. The American Statistician. 2014. № 68 (3). P. 191-195. DOI: 10.1080/00031305.2014.917055.

33. Тихонов А.Н., Арсенин В.Я. Методы решения некорректных задач. Москва : Наука, 1974.

34. Иванов В.К., Васин В.В., Танана В.П. Теория линейных некорректных задач и ее приложения. Москва : Наука, 1978.

35. Вапник В.Н., Глазкова Т.Г., Кощеев В.А., Михальский А.И., Червоненкис А.Я. Алгоритмы и программы восстановления зависимостей. Москва : Физматлит, 1984. 816 с.

36. Полянин А.Д., Манжиров А.В. Справочник по интегральным уравнениям. Москва : Физматлит, 2003.

37. Латыпов А.Ф. Численные методы решения линейных интегральных уравнений Фредгольма и Вольтерра 1-го рода. Сборник докладов Международной конференции «Обратные и некорректные задачи математической физики», посвященная 75-летию академика М.М. Лаврентьева, 20-25 августа 2007 г., Новосибирск

\section{Information about author: Kudryavtsev P. G.,}

Candidate of Chemical Sciences, Professor, Full member of the International Academy of Ecology and Life Protection Sciences (IAELPS), Full member of the Russian Academy of Natural Sciences (RANS), Deputy Director of R\&D,

Polymate Ltd - Israel Nanotechnology Research Center, Editor in Chief of the Journal "Scientific Israel-Technological Advantages" POBox 73, Migdal HaEmek 2310001, Israel 